DOI 10.4171/JEMS/224

K. Soundararajan · Matthew P. Young

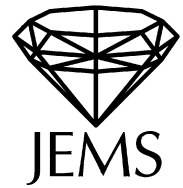

\title{
The second moment of quadratic twists of modular $L$-functions
}

Received July 27, 2009 and in revised form January 5, 2010

\begin{abstract}
We study the second moment of the central values of quadratic twists of a modular $L$-function. Unconditionally, we obtain a lower bound which matches the conjectured asymptotic formula, while on GRH we prove the asymptotic formula itself.
\end{abstract}

\section{Introduction}

The family of quadratic twists of a modular form has received much attention in recent years. Motivated by the Birch-Swinnerton-Dyer conjectures, we seek an understanding of the central values of the associated $L$-functions, and while this question has been investigated extensively, much remains unknown. One important theme in this area concerns the moments of these central $L$-values. Thanks to the work of Keating and Snaith [12] there are now widely believed conjectures for the asymptotics of such moments, but only the asymptotic for the first moment has been proved (see [2, 10, 13]). In this paper we establish two results concerning the second moment of the central $L$-values. Unconditionally we obtain a lower bound for the second moment which matches precisely the conjectured asymptotic formula. Upon assuming the truth of the Generalized Riemann Hypothesis, we establish the conjectured asymptotic formula.

To state our results we need some notation. For simplicity we shall work with modular forms of full level but our work can be extended to congruence subgroups. Let $f$ be a modular form of weight $\kappa$ for the full modular group and suppose that $f$ is an eigenfunction of all the Hecke operators. We write the Fourier expansion of $f$ as

$$
f(z)=\sum_{n=1}^{\infty} \lambda_{f}(n) n^{(\kappa-1) / 2} e(n z)
$$

with $\lambda_{f}(1)=1$, and $f$ has been normalized so that Deligne's bound gives $\left|\lambda_{f}(n)\right| \leq d(n)$ for all $n$, where $d(n)$ denotes the number of divisors of $n$. The $L$-function associated to $f$ is

K. Soundararajan: Stanford University, 450 Serra Mall, Building 380, Stanford, CA 94305-2125, U.S.A.; e-mail: ksound@math.stanford.edu

M. P. Young: Department of Mathematics, Texas A\&M University, College Station,

TX 77843-3368, U.S.A.; e-mail: myoung@ math.tamu.edu 


$$
L(s, f)=\sum_{n=1}^{\infty} \frac{\lambda_{f}(n)}{n^{s}}=\prod_{p}\left(1-\frac{\lambda_{f}(p)}{p^{s}}+\frac{1}{p^{2 s}}\right)^{-1},
$$

which converges absolutely for $\operatorname{Re}(s)>1$, extends analytically to the entire complex plane, and satisfies the functional equation

$$
\Lambda(s, f)=(2 \pi)^{-s} \Gamma\left(s+\frac{\kappa-1}{2}\right) L(s, f)=i^{\kappa} \Lambda(1-s, f) .
$$

Let $d$ denote a fundamental discriminant, and $\chi_{d}(\cdot)=\left(\frac{d}{2}\right)$ denote the primitive quadratic character of conductor $|d|$. Let $f \otimes \chi_{d}$ denote the twist of $f$ by the character $\chi_{d}$, and $L\left(s, f \otimes \chi_{d}\right)$ denote the twisted $L$-function

$$
L\left(s, f \otimes \chi_{d}\right)=\sum_{n=1}^{\infty} \frac{\lambda_{f}(n)}{n^{s}} \chi_{d}(n) .
$$

We set

$$
\Lambda\left(s, f \otimes \chi_{d}\right)=\left(\frac{|d|}{2 \pi}\right)^{s} \Gamma\left(s+\frac{\kappa-1}{2}\right) L\left(s, f \otimes \chi_{d}\right),
$$

and then the twisted $L$-function satisfies the functional equation

$$
\Lambda\left(s, f \otimes \chi_{d}\right)=i^{\kappa} \epsilon(d) \Lambda\left(1-s, f \otimes \chi_{d}\right),
$$

where $\epsilon(d)=\left(\frac{d}{-1}\right)$ is 1 or -1 depending on whether $d$ is positive or negative. Note that the sign of the functional equation is negative if $\kappa \equiv 2(\bmod 4)$ and $d$ is positive, or if $\kappa \equiv 0(\bmod 4)$ and $d$ is negative, and in these cases the central $L$-value is zero.

Below we shall use $\sum^{*}$ to denote a sum over square-free integers, and $\sum^{b}$ to denote a sum over fundamental discriminants.

Theorem 1.1. Let $\kappa \equiv 0(\bmod 4)$, and keep notations as above. Then

$$
\sum_{\substack{0<8 d \leq X \\(d, 2)=1}}^{*} L\left(1 / 2, f \otimes \chi_{8 d}\right)^{2} \geq(c+o(1)) X \log X
$$

where

$$
c=\frac{2}{\pi^{2}} L\left(1, \operatorname{sym}^{2} f\right)^{3} Z_{2}(0,0),
$$

and the value $Z_{2}(0,0)$ is defined in (4.5) and (4.6).

In Theorem 1.1, $c$ is the constant predicted by the Keating-Snaith conjectures (see [12, 5]). For simplicity we have restricted attention to fundamental discriminants of the form $8 d$, but we may also handle similarly all discriminants.

Rudnick and Soundararajan [14, 15] have described a general method to obtain lower bounds for moments in families of $L$-functions. Their method would readily give a bound $\gg X \log X$ in Theorem 1.1 .

The problem of estimating the second moment of quadratic twists of a modular form is comparable in difficulty with that of estimating the fourth moment of central values of quadratic Dirichlet $L$-functions. Analogously to Theorem 1.1 we could obtain a lower bound for that fourth moment which matches precisely the conjectured asymptotic formula; this was stated without proof in [15]. 
Theorem 1.2. Suppose the Generalized Riemann Hypothesis holds for the family of $L$-functions $L\left(s, f \otimes \chi_{d}\right)$ for all fundamental discriminants $d$, and also for $\zeta(s)$ and $L\left(s, \operatorname{sym}^{2} f\right)$. Then, for $\kappa \equiv 0(\bmod 4)$, and with $c$ being the constant in Theorem 1.1

$$
\sum_{\substack{0<8 d \leq X \\(d, 2)=1}}^{*} L\left(1 / 2, f \otimes \chi_{8 d}\right)^{2}=(c+o(1)) X \log X .
$$

Our method would allow us to get an error term in Theorem 1.2 which is smaller than the main term by a small power of $\log X$. If we consider a smooth sum over the discriminants $8 d$ in place of the "sharp cut-off" $0<8 d \leq X$ we would get an error term of $O\left(X(\log X)^{3 / 4+\varepsilon}\right)$ (see Section 5 below).

The new input in Theorem 1.2 arises from recent work of the first author [17] on obtaining upper bounds for moments of $L$-functions assuming the GRH. The work there will show that our second moment is (on GRH) bounded above by $X(\log X)^{1+\varepsilon}$. To refine this to the asymptotic given here, we need to extend the technique in [17] to bound shifted moments of $L$-functions; for a precise statement see Theorem 6.1 below. Similar upper bounds for analogous shifted moments for the Riemann zeta-function have recently been obtained by Chandee [3].

As with Theorem 1.1 for simplicity we have restricted attention to fundamental discriminants of the form $8 d$, and we may adapt our methods to cover other discriminants. In families of discriminants where the sign of the functional equation is negative, we may adapt our methods to study the second moment of the derivative of the $L$-function at $1 / 2$. Further, we may adapt the technique described here to obtain an asymptotic formula for the fourth moment of quadratic Dirichlet $L$-functions, conditional on the GRH. We note here the recent work of Bucur and Diaconu [1] which treats the fourth moment of quadratic Dirichlet $L$-functions over the rational function field.

Given two Hecke eigenforms $f$ and $g$ (with weights that are congruent modulo 4 ) it is a very interesting problem to understand averages of $L\left(1 / 2, f \otimes \chi_{d}\right) L\left(1 / 2, g \otimes \chi_{d}\right)$. An asymptotic formula or lower bound for this quantity could be used to show that there are quadratic twists for which $L\left(1 / 2, f \otimes \chi_{d}\right)$ and $L\left(1 / 2, g \otimes \chi_{d}\right)$ are both nonzero; a result that is as yet unknown. Unfortunately the methods of this paper do not shed any light on this problem.

\section{Basic tools}

In this section we gather some of the standard formulas and estimates we shall need.

\subsection{The approximate functional equation}

Let $d$ be a fundamental discriminant and let $s$ be a complex number in the critical strip. We define (for any positive $c$ )

$$
W_{w}(x):=\frac{1}{2 \pi i} \int_{(c)} \frac{\Gamma(w+(\kappa-1) / 2+s)}{\Gamma(w+(\kappa-1) / 2)}(2 \pi x)^{-s} \frac{d s}{s} .
$$


A particular case is when $w=1 / 2$ where we have

$$
W_{1 / 2}(x)=\frac{1}{2 \pi i} \int_{(c)} \frac{g(s)}{s} x^{-s} d s, \quad \text { where } g(s)=(2 \pi)^{-s} \frac{\Gamma(\kappa / 2+s)}{\Gamma(\kappa / 2)} .
$$

We also set

$$
\mathcal{A}(s, d):=\sum_{n=1}^{\infty} \frac{\lambda_{f}(n) \chi_{d}(n)}{n^{s}} W_{s}\left(\frac{n}{|d|}\right) .
$$

The function $W_{w}(x)$ decays rapidly as $x \rightarrow \infty$; this may be checked by taking $c$ suitably large in the definition of $W_{w}(x)$, and using Stirling's formula.

Lemma 2.1. With notations as above we have

$$
L\left(s, f \otimes \chi_{d}\right)=\mathcal{A}(s, d)+i^{\kappa} \epsilon(d)\left(\frac{|d|}{2 \pi}\right)^{1-2 s} \frac{\Gamma(1-s)}{\Gamma(s)} \mathcal{A}(1-s, d) .
$$

Lemma 2.1 is a standard "approximate functional equation" (see for example Theorem 5.3 of [11]). Note that if $s=1 / 2$ then $L\left(1 / 2, f \otimes \chi_{d}\right)=\left(1+i^{\kappa} \epsilon(d)\right) \mathcal{A}(1 / 2, d)$.

\subsection{Poisson summation}

We now quote Lemma 2.6 of [18].

Lemma 2.2. Let $F$ be a smooth function with compact support on the positive real numbers, and suppose that $n$ is an odd integer. Then

$$
\sum_{(d, 2)=1}\left(\frac{d}{n}\right) F\left(\frac{d}{Z}\right)=\frac{Z}{2 n}\left(\frac{2}{n}\right) \sum_{k \in \mathbb{Z}}(-1)^{k} G_{k}(n) \widehat{F}\left(\frac{k Z}{2 n}\right),
$$

where

$$
G_{k}(n)=\left(\frac{1-i}{2}+\left(\frac{-1}{n}\right) \frac{1+i}{2}\right) \sum_{a(\bmod n)}\left(\frac{a}{n}\right) e\left(\frac{a k}{n}\right),
$$

and

$$
\widehat{F}(y)=\int_{-\infty}^{\infty}(\cos (2 \pi x y)+\sin (2 \pi x y)) F(x) d x
$$

is a Fourier-type transform of $F$.

The Gauss-type sum $G_{k}(n)$ has been calculated explicitly in Lemma 2.3 of [18] which we quote below.

Lemma 2.3. If $m$ and $n$ are relatively prime odd integers, then $G_{k}(m n)=G_{k}(m) G_{k}(n)$, and if $p^{\alpha}$ is the largest power of $p$ dividing $k$ (setting $\alpha=\infty$ if $k=0$ ), then

$$
G_{k}\left(p^{\beta}\right)= \begin{cases}0 & \text { if } \beta \leq \alpha \text { is odd, } \\ \phi\left(p^{\beta}\right) & \text { if } \beta \leq \alpha \text { is even, } \\ -p^{\alpha} & \text { if } \beta=\alpha+1 \text { is even } \\ \left(\frac{k p^{-\alpha}}{p}\right) p^{\alpha} \sqrt{p} & \text { if } \beta=\alpha+1 \text { is odd } \\ 0 & \text { if } \beta \geq \alpha+2 .\end{cases}
$$




\subsection{The large sieve for quadratic characters}

Heath-Brown [9] proved the following large-sieve type inequality for quadratic characters.

Theorem 2.4. For any $M, N \geq 1$ and any sequence of complex numbers $a_{n}$, we have

$$
\sum_{\substack{m \leq M \\ m \text { odd }}}^{*}\left|\sum_{n \leq N}^{*} a_{n}\left(\frac{n}{m}\right)\right|^{2} \ll(M N)^{\varepsilon}(M+N) \sum_{n \leq N}\left|a_{n}\right|^{2} .
$$

Using the approximate functional equation of Lemma 2.1 and Heath-Brown's result we may easily deduce the following estimate (a simple modification of Theorem 2 of [9]).

Corollary 2.5. For $\sigma \geq 1 / 2$ and any $\varepsilon>0$, we have

$$
\sum_{|d| \leq X}^{b}\left|L\left(\sigma+i t, f \otimes \chi_{d}\right)\right|^{2} \ll_{\varepsilon}(X(1+|t|))^{1+\varepsilon} .
$$

\section{The main proposition}

In this section we describe the main calculation that leads to the proof of Theorem 1.1 The pattern of this proof is also followed, with a few modifications, in obtaining a stronger result leading to Theorem 1.2 , we shall describe this in Section 5

Our aim here is to establish an asymptotic formula for

$$
S(h):=\sum_{(d, 2)=1}^{*} \sum_{n_{1}} \sum_{n_{2}} \frac{\lambda_{f}\left(n_{1}\right) \lambda_{f}\left(n_{2}\right)}{\sqrt{n_{1} n_{2}}} \chi_{8 d}\left(n_{1} n_{2}\right) h\left(d, n_{1}, n_{2}\right),
$$

where $h$ is a smooth function on $\mathbb{R}_{+}^{3}$.

Proposition 3.1. Let $X, U_{1}$ and $U_{2}$ be large, and suppose that $U_{1} U_{2} \leq X^{2}$. Let $h(x, y, z)$ be a smooth function on $\mathbb{R}_{+}^{3}$ which is compactly supported in the $x$-variable, having all partial derivatives extending continuously to the boundary, and satisfying the partial derivative bounds

$$
x^{i} y^{j} z^{k} h^{(i, j, k)}(x, y, z) \ll i, j, k\left(1+\frac{x}{X}\right)^{-100}\left(1+\frac{y}{U_{1}}\right)^{-100}\left(1+\frac{z}{U_{2}}\right)^{-100}
$$

Then, setting $h_{1}(y, z)=\int_{0}^{\infty} h(x X, y, z) d x$, we have

$$
S(h)=\frac{4 X}{\pi^{2}} \sum_{\substack{\left(n_{1} n_{2}, 2\right)=1 \\ n_{1} n_{2}=\square}} \frac{\lambda_{f}\left(n_{1}\right) \lambda_{f}\left(n_{2}\right)}{\sqrt{n_{1} n_{2}}}\left(\prod_{p \mid n_{1} n_{2}} \frac{p}{p+1}\right) h_{1}\left(n_{1}, n_{2}\right)+O\left(\left(U_{1} U_{2}\right)^{1 / 4} X^{1 / 2+\varepsilon}\right) .
$$


We begin the proof of Proposition 3.1 by using Möbius inversion to remove the squarefree condition on $d$. Thus we write, for an appropriate parameter $Y$ to be chosen later,

$$
\begin{aligned}
S(h)= & \left(\sum_{\substack{a \leq Y \\
(a, 2)=1}}+\sum_{\substack{a>Y \\
(a, 2)=1}}\right) \mu(a) \\
& \times \sum_{\substack{(d, 2)=1\\
}} \sum_{\left(n_{1}, a\right)=1} \sum_{\left(n_{2}, a\right)=1} \frac{\lambda_{f}\left(n_{1}\right) \lambda_{f}\left(n_{2}\right)}{\sqrt{n_{1} n_{2}}} \chi_{8 d}\left(n_{1} n_{2}\right) h\left(d a^{2}, n_{1}, n_{2}\right) \\
= & S_{1}(h)+S_{2}(h) .
\end{aligned}
$$

\subsection{Estimating $S_{2}(h)$}

We first estimate the easier term $S_{2}(h)$.

Lemma 3.2. We have $S_{2}(h) \ll X^{1+\varepsilon} Y^{-1}$.

Proof. We write $d=b^{2} \ell$ where $\ell$ is square-free, and group terms according to $c=a b$. Thus

$$
S_{2}(h)=\sum_{(c, 2)=1} \sum_{\substack{a>Y \\ a \mid c}} \mu(a) \sum_{(\ell, 2)=1}^{*} \sum_{\left(n_{1}, c\right)=1} \sum_{\left(n_{2}, c\right)=1} \frac{\lambda_{f}\left(n_{1}\right) \lambda_{f}\left(n_{2}\right)}{\sqrt{n_{1} n_{2}}} \chi_{8 \ell}\left(n_{1} n_{2}\right) h\left(c^{2} \ell, n_{1}, n_{2}\right) .
$$

Consider the sum over $\ell, n_{1}$, and $n_{2}$ in 3.1. Using Mellin transforms in the variables $n_{1}$ and $n_{2}$ we see that this sum is

$$
\frac{1}{(2 \pi i)^{2}} \int_{(1 / 2+\varepsilon)} \int_{(1 / 2+\varepsilon)} \sum_{(\ell, 2)=1}^{*} \check{h}\left(c^{2} \ell ; u, v\right) \sum_{\substack{n_{1}, n_{2} \\\left(n_{1} n_{2}, c\right)=1}} \frac{\lambda_{f}\left(n_{1}\right) \lambda_{f}\left(n_{2}\right)}{n_{1}^{1 / 2+u} n_{2}^{1 / 2+v}} \chi_{8 \ell}\left(n_{1}\right) \chi_{8 \ell}\left(n_{2}\right) d u d v
$$

where

$$
\breve{h}(x ; u, v)=\int_{0}^{\infty} \int_{0}^{\infty} h(x, y, z) y^{u} z^{v} \frac{d y}{y} \frac{d z}{z} .
$$

Integrating by parts several times, we see that for $\operatorname{Re}(u), \operatorname{Re}(v)>0$,

$$
\breve{h}(x ; u, v) \ll\left(1+\frac{x}{X}\right)^{-100} \frac{U_{1}^{\operatorname{Re}(u)} U_{2}^{\operatorname{Re}(v)}}{|u v|(1+|u|)^{10}(1+|v|)^{10}} .
$$

The sum over $n_{1}$ and $n_{2}$ in 3.2 equals $L_{c}\left(1 / 2+u, f \otimes \chi_{8 \ell}\right) L_{c}\left(1 / 2+v, f \otimes \chi_{8 \ell}\right)$ where $L_{c}$ is given by the Euler product defining $L(s, f)$ but omitting those primes dividing $c$. Thus moving the lines of integration in 3.2 to $\operatorname{Re}(u)=\operatorname{Re}(v)=1 / \log X$, and using (3.3) together with

$$
\begin{aligned}
& \left|L_{c}\left(1 / 2+u, f \otimes \chi_{8 \ell}\right) L_{c}\left(1 / 2+v, f \otimes \chi_{8 \ell}\right)\right| \\
& \quad \leq d(c)^{2}\left(\left|L\left(1 / 2+u, f \otimes \chi_{8 \ell}\right)\right|^{2}+\left|L\left(1 / 2+v, f \otimes \chi_{8 \ell}\right)\right|^{2}\right),
\end{aligned}
$$


we conclude that $(3.2)$ is bounded by

$d(c)^{2}(\log X)^{2} \int_{-\infty}^{\infty}(1+|t|)^{-10} \sum_{(\ell, 2)=1}^{*}\left(1+\frac{\ell c^{2}}{X}\right)^{-100}\left|L\left(\frac{1}{2}+\frac{1}{\log X}+i t, f \otimes \chi_{8 \ell}\right)\right|^{2} d t$.

Now using Corollary 2.5 we conclude that the quantity in 3.2 is $\ll d(c)^{2} X^{1+\varepsilon} / c^{2}$, and using this estimate in (3.1) we obtain the lemma.

Now let us consider the harder problem of evaluating $S_{1}(h)$. We begin by applying Poisson summation, Lemma 2.2 Letting $C=\cos$ and $S=\sin$, we get

$$
\begin{aligned}
S_{1}(h)=\frac{X}{2} \sum_{\substack{a \leq Y \\
(a, 2)=1}} \frac{\mu(a)}{a^{2}} \sum_{k \in \mathbb{Z}} \sum_{\left(n_{1}, 2 a\right)=1} \sum_{\left(n_{2}, 2 a\right)=1} \frac{\lambda_{f}\left(n_{1}\right) \lambda_{f}\left(n_{2}\right)}{\sqrt{n_{1} n_{2}}} \frac{G_{k}\left(n_{1} n_{2}\right)}{n_{1} n_{2}} \\
\times \int_{0}^{\infty} h\left(x X, n_{1}, n_{2}\right)(C+S)\left(\frac{2 \pi k x X}{2 n_{1} n_{2} a^{2}}\right) d x .
\end{aligned}
$$

\subsection{The main term}

The main contribution to $S_{1}(h)$ comes from the $k=0$ term in 3.5 , which we call $S_{10}(h)$. Note $G_{0}(m)=\phi(m)$ if $m=\square$, and is zero otherwise. Further

$$
\begin{aligned}
\sum_{\substack{a \leq Y \\
\left(a, 2 n_{1} n_{2}\right)=1}} \frac{\mu(a)}{a^{2}} & =\frac{1}{\zeta(2)} \prod_{p \mid 2 n_{1} n_{2}}\left(1-\frac{1}{p^{2}}\right)^{-1}+O\left(Y^{-1}\right) \\
& =\frac{8}{\pi^{2}} \prod_{p \mid n_{1} n_{2}}\left(1-\frac{1}{p^{2}}\right)^{-1}+O\left(Y^{-1}\right) .
\end{aligned}
$$

Hence, setting $h_{1}(y, z)=\int_{0}^{\infty} h(x X, y, z) d x$ we obtain

$$
\begin{aligned}
S_{10}(h)= & \frac{4 X}{\pi^{2}} \sum_{\substack{\left(n_{1} n_{2}, 2\right)=1 \\
n_{1} n_{2}=\square}} \frac{\lambda_{f}\left(n_{1}\right) \lambda_{f}\left(n_{2}\right)}{\sqrt{n_{1} n_{2}}}\left(\prod_{p \mid n_{1} n_{2}} \frac{p}{p+1}\right) h_{1}\left(n_{1}, n_{2}\right) \\
& +O\left(\frac{X}{Y} \sum_{\substack{\left(n_{1} n_{2}, 2\right)=1 \\
n_{1} n_{2}=\square}} \frac{d\left(n_{1}\right) d\left(n_{2}\right)}{\sqrt{n_{1} n_{2}}}\left|h_{1}\left(n_{1}, n_{2}\right)\right|\right) .
\end{aligned}
$$

Using the bounds for $h$ assumed in Proposition 3.1 (which basically restrict $n_{1}$ to be of size $U_{1}$ and $n_{2}$ to be of size $U_{2}$ ) we find that the error term above is $\ll X(\log X)^{11} / Y$ so that

$$
S_{10}(h)=\frac{4 X}{\pi^{2}} \sum_{\substack{\left(n_{1} n_{2}, 2\right)=1 \\ n_{1} n_{2}=\square}} \frac{\lambda_{f}\left(n_{1}\right) \lambda_{f}\left(n_{2}\right)}{\sqrt{n_{1} n_{2}}}\left(\prod_{p \mid n_{1} n_{2}} \frac{p}{p+1}\right) h_{1}\left(n_{1}, n_{2}\right)+O\left(\frac{X}{Y}(\log X)^{11}\right) .
$$




\subsection{The $k \neq 0$ terms}

We now estimate the contribution to $S_{1}(h)$ from the terms $k \neq 0$ in (3.5); call this contribution $S_{3}(h)$.

We first express the weight function appearing in $(3.5)$ in a form more suitable for Mellin transforms. Suppose $f$ is a smooth function on $\mathbb{R}_{+}$with rapid decay at infinity, and such that $f$ and all its derivatives have a finite limit as $x \rightarrow 0^{+}$. Consider the Fourierlike transform

$$
\widehat{f_{C S}}(y):=\int_{0}^{\infty} f(x) C S(2 \pi x y) d x,
$$

where $C S$ stands for either cos or sin. By Mellin inversion, we get

$$
\begin{aligned}
\widehat{f}_{C S}(y) & =\int_{0}^{\infty} C S(2 \pi x y) \frac{1}{2 \pi i} \int_{(-1 / 2)} \tilde{f}(1+s) x^{-s} d s \frac{d x}{x} \\
& =\int_{0}^{\infty} C S(\operatorname{sgn}(y) x) \frac{1}{2 \pi i} \int_{(1 / 2)} \tilde{f}(1-s)(x / 2 \pi|y|)^{s} d s \frac{d x}{x} .
\end{aligned}
$$

Reversing the order of integrations and using $(17.43 .3,17.43 .4)$ of [8] simplifies the above to

$$
\widehat{f}_{C S}(y)=\frac{1}{2 \pi i} \int_{(1 / 2)} \tilde{f}(1-s) \Gamma(s) C S\left(\frac{\operatorname{sgn}(y) \pi s}{2}\right)(2 \pi|y|)^{-s} d s .
$$

One can rigorously justify the interchange of integrations by splitting the $x$-integral into $x \leq Z$ (with $Z$ large) and $x>Z$; one treats the integral with $x>Z$ by a contour shift, while the $x \leq Z$ integral gets interchanged with the $s$-integral, and then extended to all $x \geq 0$ by integration by parts.

Applying this formula, we have

$$
\begin{aligned}
\int_{0}^{\infty} & h\left(X x, n_{1}, n_{2}\right)(C+S)\left(\frac{2 \pi k x X}{2 n_{1} n_{2} a^{2}}\right) d x \\
\quad= & \frac{X^{-1}}{2 \pi i} \int_{(\varepsilon)} \check{h}\left(1-s ; n_{1}, n_{2}\right)\left(\frac{n_{1} n_{2} a^{2}}{\pi|k|}\right)^{s} \Gamma(s)(C+\operatorname{sgn}(k) S)\left(\frac{\pi s}{2}\right) d s,
\end{aligned}
$$

where

$$
\check{h}(s ; y, z)=\int_{0}^{\infty} h(x, y, z) x^{s} \frac{d x}{x} .
$$

Taking the Mellin transforms in the other variables on the second line of 3.7), we get

$$
\begin{aligned}
& \frac{1}{X}\left(\frac{1}{2 \pi i}\right)^{3} \\
& \quad \times \int_{(\varepsilon)} \int_{(\varepsilon)} \int_{(\varepsilon)} \tilde{h}(1-s, u, v) \frac{1}{n_{1}^{u} n_{2}^{v}}\left(\frac{n_{1} n_{2} a^{2}}{\pi|k|}\right)^{s} \Gamma(s)(C+\operatorname{sgn}(k) S)\left(\frac{\pi s}{2}\right) d s d u d v,
\end{aligned}
$$

where

$$
\widetilde{h}(s, u, v)=\int_{\mathbb{R}_{+}^{3}} h(x, y, z) x^{s} y^{u} z^{v} \frac{d x}{x} \frac{d y}{y} \frac{d z}{z} .
$$


Integrating by parts several times we find that for $\operatorname{Re}(u), \operatorname{Re}(v)>0$ we have

$$
|\tilde{h}(s, u, v)| \ll \frac{X^{\operatorname{Re}(s)} U_{1}^{\operatorname{Re}(u)} U_{2}^{\operatorname{Re}(v)}}{|u v|(1+|s|)^{98}(1+|u|)^{98}(1+|v|)^{98}} .
$$

Using this expression in 3.5 , and since $G_{k}(m)=G_{4 k}(m)$ for odd $m$, we find that

$$
\begin{aligned}
S_{3}(h) & =\frac{1}{2} \sum_{\substack{a \leq Y \\
(a, 2)=1}} \frac{\mu(a)}{a^{2}} \sum_{k \neq 0} \sum_{\left(n_{1}, 2 a\right)=1} \sum_{\left(n_{2}, 2 a\right)=1} \frac{\lambda_{f}\left(n_{1}\right) \lambda_{f}\left(n_{2}\right)}{\sqrt{n_{1} n_{2}}} \frac{G_{4 k}\left(n_{1} n_{2}\right)}{n_{1} n_{2}}\left(\frac{1}{2 \pi i}\right)^{3} \\
& \times \int_{(\varepsilon)} \int_{(\varepsilon)} \int_{(\varepsilon)} \tilde{h}(1-s, u, v) \frac{1}{n_{1}^{u} n_{2}^{v}}\left(\frac{n_{1} n_{2} a^{2}}{\pi|k|}\right)^{s} \Gamma(s)(C+\operatorname{sgn}(k) S)\left(\frac{\pi s}{2}\right) d s d u d v .
\end{aligned}
$$

We write $4 k=k_{1} k_{2}^{2}$ where $k_{1}$ is a fundamental discriminant, and $k_{2}$ is positive, so that the sum over $k$ above is a sum over fundamental discriminants $k_{1}$ and positive integers $k_{2}$. We consider the sum over $k_{2}, n_{1}$ and $n_{2}$ in (3.9) above. Note that the integrals in (3.9) may be taken over any vertical lines with real part between 0 and 1 . Consider

$$
Z\left(\alpha, \beta, \gamma ; q, k_{1}\right)=\sum_{k_{2}=1}^{\infty} \sum_{\left(n_{1}, 2 q\right)=1} \sum_{\left(n_{2}, 2 q\right)=1} \frac{\lambda_{f}\left(n_{1}\right) \lambda_{f}\left(n_{2}\right)}{n_{1}^{\alpha} n_{2}^{\beta}\left|k_{2}\right|^{2 \gamma}} \frac{G_{k_{1} k_{2}^{2}}\left(n_{1} n_{2}\right)}{n_{1} n_{2}}
$$

which converges absolutely if $\operatorname{Re}(\alpha), \operatorname{Re}(\beta)$, and $\operatorname{Re}(\gamma)$ are all $>1 / 2$. Therefore taking the integrals in 3.9 to be on the lines $\operatorname{Re}(s)=1 / 2+\varepsilon$ and $\operatorname{Re}(u)=\operatorname{Re}(v)=1 / 2+\varepsilon$ we find that

$$
\begin{aligned}
S_{3}(h) & =\frac{1}{2} \sum_{\substack{a \leq Y \\
(a, 2)=1}} \frac{\mu(a)}{a^{2}} \sum_{k_{1}}^{b}\left(\frac{1}{2 \pi i}\right)^{3} \int_{(1 / 2+2 \varepsilon)} \int_{(1 / 2+\varepsilon)} \int_{(1 / 2+\varepsilon)} \tilde{h}(1-s, u, v) \Gamma(s) \\
& \times\left(C+\operatorname{sgn}\left(k_{1}\right) S\right)\left(\frac{\pi s}{2}\right)\left(\frac{a^{2}}{\pi\left|k_{1}\right|}\right)^{s} Z\left(1 / 2+u-s, 1 / 2+v-s, s ; a, k_{1}\right) d s d u d v .
\end{aligned}
$$

Changing variables we conclude that

$$
\begin{gathered}
S_{3}(h)=\frac{1}{2} \sum_{\substack{a \leq Y \\
(a, 2)=1}} \frac{\mu(a)}{a^{2}} \sum_{k_{1}}^{b}\left(\frac{1}{2 \pi i}\right)^{3} \int_{(\varepsilon)} \int_{(\varepsilon)} \int_{(1 / 2+\varepsilon)} \tilde{h}(1-s, u+s, v+s) \Gamma(s) \\
\quad \times\left(C+\operatorname{sgn}\left(k_{1}\right) S\right)\left(\frac{\pi s}{2}\right)\left(\frac{a^{2}}{\pi\left|k_{1}\right|}\right)^{s} Z\left(1 / 2+u, 1 / 2+v, s ; a, k_{1}\right) d s d u d v
\end{gathered}
$$

To proceed further we require an analysis of the function $Z$. 
Lemma 3.3. The function $Z\left(\alpha, \beta, \gamma ; q, k_{1}\right)$ defined above may be written as

$$
\begin{aligned}
& \frac{L_{q}\left(1 / 2+\alpha, f \otimes \chi_{k_{1}}\right) L_{q}\left(1 / 2+\beta, f \otimes \chi_{k_{1}}\right)}{\zeta_{q}(1+\alpha+\beta) L_{q}\left(1+2 \alpha, \operatorname{sym}^{2} f\right) L_{q}\left(1+\alpha+\beta, \operatorname{sym}^{2} f\right) L_{q}\left(1+2 \beta, \operatorname{sym}^{2} f\right)} \\
& \times Z_{2}\left(\alpha, \beta, \gamma ; q, k_{1}\right),
\end{aligned}
$$

where $Z_{2}\left(\alpha, \beta, \gamma ; q, k_{1}\right)$ is a function uniformly bounded in the region $\operatorname{Re}(\gamma) \geq 1 / 2+\varepsilon$ and $\operatorname{Re}(\alpha), \operatorname{Re}(\beta) \geq 0$.

Proof. Inspecting Lemma 2.3 , we see that the summand of 3.10 is jointly multiplicative in terms of $n_{1}, n_{2}$, and $k_{2}$, so that $Z\left(\alpha, \beta, \gamma ; q, k_{1}\right)$ may be expressed as a product over all primes $p$. We must compute the contribution of such an Euler factor at $p$.

Consider first the generic case when $p \nmid 2 q k_{1}$. The contribution of such an Euler factor is

$$
\sum_{k_{2}, n_{1}, n_{2}} \frac{\lambda_{f}\left(p^{n_{1}}\right) \lambda_{f}\left(p^{n_{2}}\right)}{p^{n_{1} \alpha+n_{2} \beta+2 k_{2} \gamma}} \frac{G_{k_{1}} p^{2 k_{2}}\left(p^{n_{1}+n_{2}}\right)}{p^{n_{1}+n_{2}}} .
$$

In the region $\operatorname{Re}(\gamma) \geq 1 / 2+\varepsilon, \operatorname{Re}(\alpha), \operatorname{Re}(\beta) \geq 0$ we check using Lemma 2.3 that the terms $k_{2} \geq 1$ contribute terms of size $\ll 1 / p^{\overline{1+2 \varepsilon}}$. This leaves the contribution of the term $k_{2}=0$ which is $1+\chi_{k_{1}}(p) \lambda_{f}(p)\left(p^{-1 / 2-\alpha}+p^{-1 / 2-\beta}\right)$. From this calculation we see that this Euler factor for $Z$ matches the corresponding Euler factor in the alternative expression given in our lemma.

Next consider the case $p \mid k_{1}$ but $p \nmid 2 q$. Using Lemma 2.3 we find that in the region $\operatorname{Re}(\gamma) \geq 1 / 2+\varepsilon$ and $\operatorname{Re}(\alpha), \operatorname{Re}(\beta) \geq 0$ this Euler factor equals

$$
1-\lambda_{f}\left(p^{2}\right)\left(\frac{1}{p^{1+2 \alpha}}+\frac{1}{p^{1+\alpha+\beta}}+\frac{1}{p^{1+2 \beta}}\right)-\frac{1}{p^{1+\alpha+\beta}}+O\left(\frac{1}{p^{1+\varepsilon}}\right) .
$$

Again this matches the corresponding Euler factor prescribed in our lemma.

Finally, if $p \mid 2 q$ the corresponding Euler factor is $\left.1-p^{-2 \gamma}\right)^{-1}=1+O\left(1 / p^{1+2 \varepsilon}\right)$. With these computations we have verified the lemma.

With this information about $Z$ at hand, we return to (3.11). We split that sum into two terms based on whether $\left|k_{1}\right| \leq U_{1} U_{2} Y^{2} / X$, or not. For the first category of terms we move the lines of integration to $\operatorname{Re}(s)=3 / 4, \operatorname{Re}(u)=\operatorname{Re}(v)=-1 / 2+1 / \log X$, and for the second category we move the lines of integration to $\operatorname{Re}(s)=5 / 4, \operatorname{Re}(u)=\operatorname{Re}(v)=$ $-1 / 2+1 / \log X$. In either case we find by Lemma 3.3 that

$$
Z\left(1 / 2+u, 1 / 2+v, s ; a, k_{1}\right) \ll\left|L_{a}\left(1+u, f \otimes \chi_{k_{1}}\right) L_{a}\left(1+v, f \otimes \chi_{k_{1}}\right)\right|(\log X)^{4},
$$

which is

$$
\ll(\log X)^{4} \prod_{p \mid a}(1+10 / \sqrt{p})\left(\left|L\left(1+u, f \otimes \chi_{k_{1}}\right)\right|^{2}+\left|L\left(1+v, f \otimes \chi_{k_{1}}\right)\right|^{2}\right) .
$$


Using (3.8), 3.12), the estimate $\left|\Gamma(s)\left(C+\operatorname{sgn}\left(k_{1}\right) S\right)(\pi s / 2)\right| \ll|s|^{\operatorname{Re}(s)-1 / 2}$, and the symmetry in $u$ and $v$ we find that our first category of terms contributes

$$
\begin{aligned}
& \ll\left(X U_{1} U_{2}\right)^{1 / 4}(\log X)^{4} \sum_{a \leq Y} \frac{1}{\sqrt{a}} \prod_{p \mid a}(1+10 / \sqrt{p}) \int_{(3 / 4)} \int_{(-1 / 2+1 / \log X)} \int_{(-1 / 2+1 / \log X)} \\
& \times \sum_{\left|k_{1}\right| \leq U_{1} U_{2} Y^{2} / X}^{b} \frac{1}{\left|k_{1}\right|^{3 / 4}}\left|L\left(1+u, f \otimes \chi_{k_{1}}\right)\right|^{2} \frac{d u d v d s}{(1+|s|)^{50}(1+|u+s|)^{50}(1+|v+s|)^{50}} .
\end{aligned}
$$

Using Corollary 2.5 we conclude that the above is $\ll\left(U_{1} U_{2}\right)^{1 / 2} Y X^{\varepsilon}$.

Similarly the contribution of the second category of terms is

$$
\begin{aligned}
& \ll\left(U_{1} U_{2}\right)^{3 / 4} X^{-1 / 4}(\log X)^{4} \sum_{a \leq Y} \sqrt{a} \prod_{p \mid a}(1+10 / \sqrt{p}) \int_{(5 / 4)} \int_{(-1 / 2+1 / \log X)} \int_{(-1 / 2+1 / \log X)} \\
& \times \sum_{\left|k_{1}\right|>U_{1} U_{2} Y^{2} / X}^{b} \frac{1}{\left|k_{1}\right|^{5 / 4}}\left|L\left(1+u, f \otimes \chi_{k_{1}}\right)\right|^{2} \frac{d u d v d s}{(1+|s|)^{50}(1+|u+s|)^{50}(1+|v+s|)^{50}} .
\end{aligned}
$$

Using Corollary 2.5 again we see that the above is once again $\ll\left(U_{1} U_{2}\right)^{1 / 2} Y X^{\varepsilon}$.

Proposition 3.1 follows upon combining the work of the previous sections, choosing $Y=X^{1 / 2}\left(U_{1} U_{2}\right)^{-1 / 4}$.

\section{The lower bound: Proof of Theorem 1.1}

Let $F$ be a smooth, nonnegative, compactly supported function on $\mathbb{R}_{+}$. Define

$$
\mathcal{A}_{U}(1 / 2 ; 8 d)=2 \sum_{n=1}^{\infty} \frac{\lambda_{f}(n) \chi_{8 d}(n)}{\sqrt{n}} W\left(\frac{n}{U}\right),
$$

where $W(x)=W_{1 / 2}(x)$, and $U \leq X$ is a parameter that we shall choose shortly. Since $F$ is nonnegative, by Cauchy's inequality we have

$$
\begin{aligned}
\sum_{(d, 2)=1}^{*} L\left(1 / 2, f \otimes \chi_{8 d}\right)^{2} F(8 d / X) & \\
& \geq \frac{\left(\sum_{(d, 2)=1}^{*} L\left(1 / 2, f \otimes \chi_{8 d}\right) \mathcal{A}_{U}(1 / 2 ; 8 d) F(8 d / X)\right)^{2}}{\sum_{(d, 2)=1}^{*} \mathcal{A}_{U}(1 / 2 ; 8 d)^{2} F(8 d / X)}
\end{aligned}
$$

Write the right hand side above as $(4 A)^{2} / 4 B$, say. Using Proposition 3.1 we shall be able to evaluate $A$ and $B$ asymptotically in the range $U \leq X^{1-\varepsilon}$. If we choose $U=X^{1-\varepsilon}$ then both $4 A$ and $4 B$ will be close to the expected asymptotic for the second moment, giving the lower bound of Theorem 1.1. A similar truncation argument appeared in [16]. 
Both $A$ and $B$ may be written in a form suitable for applying Proposition 3.1. For example, we have

$$
A=\sum_{(d, 2)=1}^{*} \sum_{n_{1}} \sum_{n_{2}} \frac{\lambda_{f}\left(n_{1}\right) \lambda_{f}\left(n_{2}\right) \chi_{8 d}\left(n_{1} n_{2}\right)}{\sqrt{n_{1} n_{2}}} h\left(d, n_{1}, n_{2}\right),
$$

where $h(x, y, z)=F(8 x / X) W(y / U) W(z /(8 x))$, which satisfies the hypothesis in Proposition 3.1 with $X=X, U_{1}=U$ and $U_{2}=X$. A similar expression holds for $B$ with $h(x, y, z)=F(8 x / X) W(y / U) W(z / U)$ meeting the condition in Proposition 3.1 with $X=X, U_{1}=U_{2}=U$.

If $U \leq X^{1-\varepsilon}$ then applying Proposition 3.1 in 4.2 we find that

$$
A=\frac{4 X}{\pi^{2}} \sum_{\substack{\left(n_{1} n_{2}, 2\right)=1 \\ n_{1} n_{2}=\square}} \frac{\lambda_{f}\left(n_{1}\right) \lambda_{f}\left(n_{2}\right)}{\sqrt{n_{1} n_{2}}}\left(\prod_{p \mid n_{1} n_{2}} \frac{p}{p+1}\right) \int_{0}^{\infty} h\left(x X, n_{1}, n_{2}\right) d x+o(X) \text {. }
$$

Using the definition of $W(x)$ we obtain

$$
\int_{0}^{\infty} h\left(x X, n_{1}, n_{2}\right) d x=\frac{1}{(2 \pi i)^{2}} \int_{(1)} \int_{(1)} \frac{g(u) g(v)}{u v} \frac{U^{u} X^{v}}{n_{1}^{u} n_{2}^{v}} \frac{\tilde{F}(1+v)}{8} d u d v,
$$

where $\tilde{F}(1+v)=\int_{0}^{\infty} x^{v} F(x) d x$. Using this in (4.3) and setting

$$
Z(u, v)=\sum_{\substack{\left(n_{1} n_{2}, 2\right)=1 \\ n_{1} n_{2}=\square}} \frac{\lambda_{f}\left(n_{1}\right) \lambda_{f}\left(n_{2}\right)}{n_{1}^{1 / 2+u} n_{2}^{1 / 2+v}} \prod_{p \mid n_{1} n_{2}} \frac{p}{p+1},
$$

we conclude that

$$
A=\frac{X}{2 \pi^{2}} \frac{1}{(2 \pi i)^{2}} \int_{(1)} \int_{(1)} \frac{g(u) g(v)}{u v} U^{u} X^{v} \tilde{F}(1+v) Z(u, v) d v d u+o(X) .
$$

A simple calculation shows that $Z(u, v)$ equals

$$
\begin{aligned}
\prod_{p>2}(1+ & \frac{p}{p+1}\left[\frac{1}{2}\left(1-\frac{\lambda_{f}(p)}{p^{1 / 2+u}}+\frac{1}{p^{1+2 u}}\right)^{-1}\left(1-\frac{\lambda_{f}(p)}{p^{1 / 2+v}}+\frac{1}{p^{1+2 v}}\right)^{-1}\right. \\
& \left.\left.+\frac{1}{2}\left(1+\frac{\lambda_{f}(p)}{p^{1 / 2+u}}+\frac{1}{p^{1+2 u}}\right)^{-1}\left(1+\frac{\lambda_{f}(p)}{p^{1 / 2+v}}+\frac{1}{p^{1+2 v}}\right)^{-1}-1\right]\right) .
\end{aligned}
$$

The Euler product above converges absolutely when $\operatorname{Re}(u)$ and $\operatorname{Re}(v)$ are positive. We write

$$
\begin{aligned}
Z(u, v)= & \zeta(1+u+v) L\left(1+2 u, \operatorname{sym}^{2}(f)\right) L\left(1+2 v, \operatorname{sym}^{2}(f)\right) \\
& \times L\left(1+u+v, \operatorname{sym}^{2}(f)\right) Z_{2}(u, v),
\end{aligned}
$$

where $Z_{2}(u, v)$ converges absolutely in the region of $\operatorname{Re}(u)$ and $\operatorname{Re}(v)$ larger than $-1 / 4$ $+\varepsilon$, and is uniformly bounded there. 
We now use these observations to evaluate the double integral in (4.4). First we move the integrals there to $\operatorname{Re}(u)=\operatorname{Re}(v)=1 / 10$; no poles are encountered in this shift. Then we move the line of integration in $v$ to $\operatorname{Re}(v)=-1 / 5$. In doing so we encounter simple poles at $v=0$ and $v=-u$ whose residues we next calculate; the integrals on $\operatorname{Re}(u)=1 / 10, \operatorname{Re}(v)=-1 / 5$ are easily seen to be $O\left(X^{-1 / 10+\varepsilon}\right)$. The contribution from the residue at $v=-u$ is

$$
\begin{array}{r}
\frac{1}{2 \pi i} \int_{(1 / 10)} \frac{g(u) g(-u)}{-u^{2}} U^{u} X^{-u} L\left(1+2 u, \operatorname{sym}^{2} f\right) L\left(1-2 u, \operatorname{sym}^{2} f\right) L\left(1, \operatorname{sym}^{2} f\right) \\
\times Z_{2}(u,-u) \tilde{F}(1-u) d u
\end{array}
$$

which is $O(1)$ since $U \leq X$. Finally consider the contribution of the residue at $v=0$, namely

$$
\begin{array}{r}
\frac{1}{2 \pi i} \int_{(1 / 10)} \frac{g(u)}{u} U^{u} \zeta(1+u) L\left(1+2 u, \operatorname{sym}^{2} f\right) L\left(1+u, \operatorname{sym}^{2} f\right) L\left(1, \operatorname{sym}^{2} f\right) \\
\times Z_{2}(u, 0) \tilde{F}(1) d u .
\end{array}
$$

We now move the line of integration in $u$ to $\operatorname{Re}(u)=-1 / 5$, encountering a double pole at $u=0$, and the integral on the $-1 / 5$ line contributes $\ll U^{-1 / 5} X^{\varepsilon}$. The residue of the double pole at $u=0$ is easily seen to be

$$
\tilde{F}(1) L\left(1, \operatorname{sym}^{2} f\right)^{3} Z_{2}(0,0) \log U+O(1) .
$$

Using these observations in 4.4 we conclude that

$$
A=\frac{X}{2 \pi^{2}}\left(\tilde{F}(1) L\left(1, \operatorname{sym}^{2} f\right)^{3} Z_{2}(0,0) \log U+O(1)\right) .
$$

A similar argument shows that the same asymptotic holds for $B$. Choosing $U=X^{1-\varepsilon}$, and letting $F$ approximate the indicator function of $[0,1]$, we deduce Theorem 1.1 from the inequality 4.1 .

\section{The asymptotic on GRH: Proof of Theorem 1.2}

In this section we prove Theorem 1.2 . The key ingredient in the proof is the following upper bound on shifted moments whose proof we postpone to the next section.

Corollary 5.1. Assume GRH for the family of quadratic twists of $f$, for the Riemann zeta-function, and for the symmetric square $L$-function $L\left(s, \operatorname{sym}^{2} f\right)$. Let $t_{1}$ and $t_{2}$ be real numbers with $\left|t_{1}\right|,\left|t_{2}\right| \leq X$ and let $1 / 2 \leq \sigma \leq 1 / 2+1 / \log X$. Then

$$
\begin{aligned}
\sum_{|d| \leq x}^{b} \mid L\left(\sigma+i t_{1}, f \otimes \chi_{d}\right) L & \left(\sigma+i t_{2}, f \otimes \chi_{d}\right) \mid \\
& \ll X(\log X)^{1 / 2+\varepsilon}\left(1+\min \left((\log X)^{1 / 2},\left|t_{1}-t_{2}\right|^{-1 / 2}\right)\right) .
\end{aligned}
$$


Let $F$ be a smooth, nonnegative, compactly supported function on $\mathbb{R}_{+}$. Recall from the previous section the definition of $\mathcal{A}_{U}(1 / 2 ; 8 d)$, and write $L\left(1 / 2, f \otimes \chi_{8 d}\right)=$ $\mathcal{A}_{U}(1 / 2 ; 8 d)+\mathcal{B}_{U}(1 / 2 ; 8 d)$. We shall prove that, on GRH, for $U \leq X /(\log X)^{100}$ we have

$$
\begin{aligned}
\sum_{(d, 2)=1}^{*}\left|\mathcal{A}_{U}(1 / 2 ; 8 d)\right|^{2} F(8 d / X) & =\frac{2 X}{\pi^{2}}\left(\tilde{F}(1) L\left(1, \operatorname{sym}^{2} f\right)^{3} Z_{2}(0,0) \log U+O(1)\right), \\
\sum_{(d, 2)=1}^{*}\left|\mathcal{B}_{U}(1 / 2 ; 8 d)\right|^{2} F(8 d / X) & =O\left(X(\log X)^{1 / 2+\varepsilon}(\log (X / U))^{2}\right) .
\end{aligned}
$$

Once these two estimates are established, Theorem 1.2 will follow upon choosing $U=$ $X /(\log X)^{100}$ since

$$
\begin{aligned}
\sum_{(d, 2)=1}^{*} \mid L(1 / 2, f & \left.\otimes \chi_{8 d}\right)\left.\right|^{2} F(8 d / X)=\sum_{(d, 2)=1}^{*}\left|\mathcal{A}_{U}(1 / 2 ; 8 d)\right|^{2} F(8 d / X) \\
& +O\left(\sum_{(d, 2)=1}^{*}\left(\left|\mathcal{A}_{U}(1 / 2 ; 8 d) \mathcal{B}_{U}(1 / 2 ; 8 d)\right|+\left|\mathcal{B}_{U}(1 / 2 ; 8 d)\right|^{2}\right) F(8 d / X)\right)
\end{aligned}
$$

and using (5.1) and 5.2) together with Cauchy-Schwarz we see that the remainder term above is $O\left(X(\log X)^{3 / 4+\varepsilon}\right)$.

It remains now to prove (5.1) and (5.2). We start with the latter. From the definition of $\mathcal{B}_{U}$ we find that

$$
\mathcal{B}_{U}(1 / 2 ; 8 d)=\frac{1}{\pi i} \int_{(c)} g(s) L\left(1 / 2+s, f \otimes \chi_{8 d}\right)\left(\frac{(8 d)^{s}-U^{s}}{s}\right) d s .
$$

Since $\left((8 d)^{s}-U^{s}\right) / s$ is analytic for all $s$ we may move the line of integration above to the line $\operatorname{Re}(s)=0$, and since $\left|(8 d)^{s}-U^{s}\right| /|s| \ll|\log (8 d / U)|$ we find that

$$
\left|\mathcal{B}_{U}(1 / 2 ; 8 d)\right| \ll|\log (8 d / U)| \int_{-\infty}^{\infty}|g(i t)|\left|L\left(1 / 2+i t, f \otimes \chi_{8 d}\right)\right| d t .
$$

Therefore we find that the RHS in (5.2) is

$$
\begin{aligned}
\ll(\log X / U)^{2} \int_{-\infty}^{\infty} \int_{-\infty}^{\infty} & \left|g\left(i t_{1}\right) g\left(i t_{2}\right)\right| \\
& \times \sum_{(d, 2)=1}^{*}\left|L\left(1 / 2+i t_{1}, f \otimes \chi_{8 d}\right) L\left(1 / 2+i t_{2}, f \otimes \chi_{8 d}\right)\right| d t_{1} d t_{2} .
\end{aligned}
$$

If $\left|t_{1}\right|$ and $\left|t_{2}\right|$ are both below $X / 2$ then we use Corollary 5.1 to bound the inner sum over $d$ above. In the remaining case we use Cauchy's inequality and the bound of Corollary 2.5 Since $|g(t)|$ decreases exponentially in $|t|$, an easy calculation then gives 5.2 .

Now we turn to (5.1). The argument follows the pattern laid out in Sections 3 and 4 the results there may be improved by appealing to the estimates of Corollary 5.1 in place of the weaker estimate in Corollary 2.5. Consider Proposition 3.1, on GRH we claim that 
the remainder term there can be replaced with $O\left(\left(U_{1} U_{2}\right)^{1 / 4} X^{1 / 2}(\log X)^{20}\right)$. We give only the changes that need to be made to the argument there. In Section 3.1 we use Corollary 5.1 in place of Corollary 2.5 (in the range $|t| \leq X$ ) to estimate the quantity in (3.4). This shows that the bound in Lemma 3.2 may be replaced with $X(\log X){ }^{10} Y^{-1}$. Similarly in Section 3.3, in estimating (3.13) and 3.14) we again invoke Corollary 5.1 (in the range $|u| \leq X)$ to obtain there the improved bound of $\left(U_{1} U_{2}\right)^{1 / 2} Y(\log X)^{5}$. Choosing $Y=X^{1 / 2}\left(U_{1} U_{2}\right)^{-1 / 4}$ as before, we obtain the stated bound for the remainder term in Proposition 3.1 The argument of $\$ 4$ now goes through verbatim, establishing [5.1.

\section{Upper bounds for shifted moments assuming GRH}

Given a real number $x \geq 10$ and a complex number $z$, set

$$
\mathcal{L}(z, x)= \begin{cases}\log \log x, & |z| \leq(\log x)^{-1} \\ -\log |z|, & (\log x)^{-1} \leq|z| \leq 1, \\ 0, & 1 \leq|z|\end{cases}
$$

If $z_{1}$ and $z_{2}$ are complex numbers we define

$$
\mathcal{M}\left(z_{1}, z_{2}, x\right)=-\frac{1}{2}\left(\mathcal{L}\left(z_{1}, x\right)+\mathcal{L}\left(z_{2}, x\right)\right),
$$

and

$$
\begin{aligned}
\mathcal{V}\left(z_{1}, z_{2}, x\right)= & \frac{1}{2}\left(\mathcal{L}\left(2 z_{1}, x\right)+\mathcal{L}\left(2 z_{2}, x\right)+\mathcal{L}\left(2 \operatorname{Re} z_{1}, x\right)+\mathcal{L}\left(2 \operatorname{Re} z_{2}, x\right)\right. \\
& \left.+2 \mathcal{L}\left(z_{1}+z_{2}, x\right)+2 \mathcal{L}\left(z_{1}+\overline{z_{2}}, x\right)\right) .
\end{aligned}
$$

This section is devoted to establishing, on GRH, the following estimates for shifted moments of $L$-functions. An immediate consequence of this theorem is Corollary 5.1 which we used above to establish Theorem 1.2 .

Theorem 6.1. Let $X$ be large, and let $z_{1}$ and $z_{2}$ be two complex numbers with $0 \leq$ $\operatorname{Re}\left(z_{1}\right), \operatorname{Re}\left(z_{2}\right) \leq 1 / \log X$ and with $\left|z_{1}\right|,\left|z_{2}\right| \leq X$. Assume GRH for the family of quadratic twists of $f$, for the Riemann zeta-function, and for the symmetric square $L$ function $L\left(s, \operatorname{sym}^{2} f\right)$. Then for any positive real number $k$ and any $\varepsilon>0$ we have

$$
\begin{aligned}
& \sum_{|d| \leq X}^{b}\left|L\left(1 / 2+z_{1}, f \otimes \chi_{d}\right) L\left(1 / 2+z_{2}, f \otimes \chi_{d}\right)\right|^{k} \\
& \ll_{k, \varepsilon} X(\log X)^{\varepsilon} \exp \left(k \mathcal{M}\left(z_{1}, z_{2}, X\right)+\frac{k^{2}}{2} \mathcal{V}\left(z_{1}, z_{2}, X\right)\right) .
\end{aligned}
$$

As remarked earlier, this result follows upon modifying the method of [17], and similar results for the Riemann zeta-function were obtained by V. Chandee [3]. If we set $z_{1}=$ $z_{2}=i t$ for a real number $t$, then it is expected that when $t$ is close to zero the moments correspond to a family with "orthogonal" symmetry, while for larger $t$ (for example $t=1$ ) the expected symmetry type is "unitary." We note that our theorem above expresses in a uniform way the transition between these symmetry types. 
To prove Theorem 6.1, we shall establish an estimate on the frequency with which large values of $\left|L\left(1 / 2+z_{1}, f \otimes \chi_{d}\right) L\left(1 / 2+z_{2}, f \otimes \chi_{d}\right)\right|$ are attained. As $d$ varies over the discriminants of size below $X$ we expect that $\log \mid L\left(1 / 2+z_{1}, f \otimes \chi_{d}\right) L\left(1 / 2+z_{2}\right.$, $\left.f \otimes \chi_{d}\right) \mid$ is distributed normally with mean $\mathcal{M}\left(z_{1}, z_{2}, X\right)$ and variance $\mathcal{V}\left(z_{1}, z_{2}, X\right)$. The next proposition establishes (in a range sufficient to prove Theorem 6.1) an upper bound for the frequency of large values that conforms to the above prediction. In what follows it may be helpful to keep in mind that for $z_{1}$ and $z_{2}$ as in Theorem 6.1 the quantity $\mathcal{V}\left(z_{1}, z_{2}, X\right)$ lies between $\log \log X+O(1)$ and $4 \log \log X+O(1)$.

Proposition 6.2. With assumptions as in Theorem 6.1 let $\mathcal{N}\left(V ; z_{1}, z_{2}, X\right)$ denote the number of fundamental discriminants $|d| \leq X$ such that

$$
\log \left|L\left(1 / 2+z_{1}, f \otimes \chi_{d}\right) L\left(1 / 2+z_{2}, f \otimes \chi_{d}\right)\right| \geq V+\mathcal{M}\left(z_{1}, z_{2}, X\right) .
$$

In the range $10 \sqrt{\log \log X} \leq V \leq \mathcal{V}\left(z_{1}, z_{2}, X\right)$ we have

$$
\mathcal{N}\left(V ; z_{1}, z_{2}, X\right) \ll X \exp \left(-\frac{V^{2}}{2 \mathcal{V}\left(z_{1}, z_{2}, X\right)}\left(1-\frac{25}{\log \log \log X}\right)\right) ;
$$

for $\mathcal{V}\left(z_{1}, z_{2}, X\right)<V \leq \frac{1}{16} \mathcal{V}\left(z_{1}, z_{2}, X\right) \log \log \log X$ we have

$$
\mathcal{N}\left(V ; z_{1}, z_{2}, X\right) \ll X \exp \left(-\frac{V^{2}}{2 \mathcal{V}\left(z_{1}, z_{2}, X\right)}\left(1-\frac{15 V}{\mathcal{V}\left(z_{1}, z_{2}, X\right) \log \log \log X}\right)^{2}\right)
$$

finally, for $\frac{1}{16} \mathcal{V}\left(z_{1}, z_{2}, X\right) \log \log \log X<V$ we have

$$
\mathcal{N}\left(V ; z_{1}, z_{2}, X\right) \ll X \exp \left(-\frac{1}{1025} V \log V\right) .
$$

We now show how Theorem 6.1 may be deduced from Proposition 6.2

Proof of Theorem 6.1. We have

$$
\begin{aligned}
\sum_{|d| \leq X}^{b} \mid L\left(1 / 2+z_{1}, f \otimes\right. & \left.\chi_{d}\right)\left.L\left(1 / 2+z_{2}, f \otimes \chi_{d}\right)\right|^{k} \\
= & -\int_{-\infty}^{\infty} \exp \left(k V+k \mathcal{M}\left(z_{1}, z_{2}, X\right)\right) d \mathcal{N}\left(V ; z_{1}, z_{2}, X\right) \\
= & k \int_{-\infty}^{\infty} \exp \left(k V+k \mathcal{M}\left(z_{1}, z_{2}, X\right)\right) \mathcal{N}\left(V ; z_{1}, z_{2}, X\right) d V
\end{aligned}
$$

Inserting here the bounds for $\mathcal{N}\left(V ; z_{1}, z_{2}, X\right)$ furnished by Proposition 6.2 we obtain Theorem 6.1 with a little calculation. This calculation may be facilitated by using Proposition 6.2 in the crude form $\mathcal{N}\left(V ; z_{1}, z_{2}, X\right) \ll X(\log X)^{o(1)} \exp \left(-V^{2} /\left(2 \mathcal{V}\left(z_{1}, z_{2}, X\right)\right)\right)$ for $3 \leq V \leq 4 k \mathcal{V}\left(z_{1}, z_{2}, X\right)$ and that $\mathcal{N}\left(V ; z_{1}, z_{2}, X\right) \ll X(\log X)^{o(1)} \exp (-4 k V)$ for larger $V$. 
It remains now to prove Proposition 6.2 We first obtain an auxiliary result analogous to the Proposition of [17], namely (6.5) below. Write $\lambda_{f}(p)=\alpha_{p}+\beta_{p}$ where $\alpha_{p} \beta_{p}=1$ and, by Deligne's theorem, $\left|\alpha_{p}\right|=\left|\beta_{p}\right|=1$. By modifying slightly the proof of the Proposition in [17] we find that for $z_{1}, z_{2}, d$ as in Theorem 6.1] and for any $2 \leq x \leq X$,

$$
\begin{aligned}
\log \mid L\left(1 / 2+z_{1}, f\right. & \left.\otimes \chi_{d}\right) L\left(1 / 2+z_{2}, f \otimes \chi_{d}\right) \mid \\
\leq & \operatorname{Re}\left(\sum_{\substack{p^{l} \leq x \\
l \geq 1}} \frac{\chi_{d}\left(p^{l}\right)\left(\alpha_{p}^{l}+\beta_{p}^{l}\right)}{l p^{l\left(1 / 2+\lambda_{0} / \log x\right)}}\left(p^{-l z_{1}}+p^{-l z_{2}}\right) \frac{\log \left(x / p^{l}\right)}{\log x}\right) \\
& +2\left(1+\lambda_{0}\right) \frac{\log X}{\log x}+O\left(\frac{1}{\log x}\right),
\end{aligned}
$$

where $\lambda_{0}=0.4912 \ldots$ is the unique real number satisfying $e^{-\lambda_{0}}=\lambda_{0}+\lambda_{0}^{2} / 2$. As in [17], the terms with $l \geq 3$ give $O(1)$. Using $\alpha_{p}^{2}+\beta_{p}^{2}=\lambda_{f}\left(p^{2}\right)-1$ and $\sum_{p \mid d} 1 / p \ll$ $\log \log \log X$ we find that the terms with $l=2$ give

$$
\operatorname{Re} \sum_{p \leq \sqrt{x}} \frac{\lambda_{f}\left(p^{2}\right)-1}{2 p^{1+2 \lambda_{0} / \log x}}\left(p^{-2 z_{1}}+p^{-2 z_{2}}\right) \frac{\log \left(x / p^{2}\right)}{\log x}+O(\log \log \log X) .
$$

Using the GRH for $L\left(s, \operatorname{sym}^{2} f\right)$ we may see that

$$
\sum_{p \leq y}\left(p^{-2 z_{1}}+p^{-2 z_{2}}\right) \lambda_{f}\left(p^{2}\right) \log p \ll \sqrt{y}(\log X y)^{2},
$$

and also the sum is trivially $\ll y$. From these bounds and partial summation we obtain

$$
\sum_{p \leq \sqrt{x}} \frac{\lambda_{f}\left(p^{2}\right)}{p^{1+2 \lambda_{0} / \log x}}\left(p^{-2 z_{1}}+p^{-2 z_{2}}\right) \frac{\log \left(x / p^{2}\right)}{\log x}=O(\log \log \log X) .
$$

Similarly RH gives that

$$
\sum_{p \leq y}\left(p^{-2 z_{1}}+p^{-2 z_{2}}\right) \log p=\frac{y^{1-2 z_{1}}}{1-2 z_{1}}+\frac{y^{1-2 z_{2}}}{1-2 z_{2}}+O\left(\sqrt{y}(\log X y)^{2}\right),
$$

and once again the sum is also trivially $\ll y$. Partial summation now shows that $-1 / 2 \sum_{p \leq \sqrt{x}} \frac{1}{p^{1+2 \lambda_{0} / \log x}}\left(p^{-2 z_{1}}+p^{-2 z_{2}}\right) \frac{\log \left(x / p^{2}\right)}{\log x}=\mathcal{M}\left(z_{1}, z_{2}, x\right)+O(\log \log \log X)$.

Inserting the above estimates into 6.2) and (6.1), and since $\mathcal{M}\left(z_{1}, z_{2}, x\right) \leq \mathcal{M}\left(z_{1}, z_{2}, X\right)$ $+\log X / \log x$, we conclude that

$$
\begin{aligned}
\log \mid L\left(1 / 2+z_{1}, f \otimes \chi_{d}\right) & L\left(1 / 2+z_{2}, f \otimes \chi_{d}\right) \mid \\
\leq & \operatorname{Re} \sum_{2<p \leq x} \frac{\lambda_{f}(p) \chi_{d}(p)}{p^{1 / 2+\lambda_{0} / \log x}\left(p^{-z_{1}}+p^{-z_{2}}\right) \frac{\log (x / p)}{\log x}} \\
& +\mathcal{M}\left(z_{1}, z_{2}, X\right)+4 \frac{\log X}{\log x}+O(\log \log \log X) .
\end{aligned}
$$


Lemma 6.3. Let $X$ and $y$ be real numbers and $k$ a natural number with $y^{k} \leq$ $X^{1 / 2} / \log X$. For any complex numbers $a(p)$ we have

$$
\sum_{|d| \leq X}^{b}\left|\sum_{2<p \leq y} \frac{a(p) \chi_{d}(p)}{p^{1 / 2}}\right|^{2 k} \ll X \frac{(2 k) !}{k ! 2^{k}}\left(\sum_{p \leq y} \frac{|a(p)|^{2}}{p}\right)^{k},
$$

where the implied constant is absolute.

Proof. Expanding out and using the Pólya-Vinogradov inequality, we have

$$
\begin{aligned}
\sum_{|d| \leq X}^{b}\left|\sum_{2<p \leq y} \frac{a(p) \chi_{d}(p)}{p^{1 / 2}}\right|^{2 k} \leq \sum_{|d| \leq X}\left|\sum_{2<p_{1}, \ldots, p_{k} \leq y} \frac{a\left(p_{1}\right) \ldots a\left(p_{k}\right)}{\sqrt{p_{1} \ldots p_{k}}}\left(\frac{d}{p_{1} \cdots p_{k}}\right)\right|^{2} \\
\ll \sum_{\substack{p_{1}, \ldots, p_{2 k} \leq y \\
p_{1} \ldots p_{2 k}=\square}} \frac{\left|a\left(p_{1}\right) \ldots a\left(p_{2 k}\right)\right|}{\sqrt{p_{1} \ldots p_{2 k}}}+O\left(\sum_{p_{1}, \ldots, p_{2 k}}\left|a\left(p_{1}\right) \ldots a\left(p_{2 k}\right) \log \left(y^{2 k}\right)\right|\right) .
\end{aligned}
$$

Since $y^{2 k} \log \left(y^{2 k}\right) \ll X$ we see, using Cauchy-Schwarz, that the second term above is

$$
\ll \log \left(y^{2 k}\right)\left(\sum_{p \leq y}|a(p)|\right)^{2 k} \leq \log \left(y^{2 k}\right)\left(\sum_{p \leq y} \frac{|a(p)|^{2}}{p}\right)^{k}\left(\sum_{p \leq y} p\right)^{k} \leq X\left(\sum_{p \leq y} \frac{|a(p)|^{2}}{p}\right)^{k} .
$$

To estimate the first term, note that $p_{1} \ldots p_{2 k}=\square$ precisely when there is a way to pair up the indices so that the corresponding primes are equal. There are $(2 k) ! /\left(k ! 2^{k}\right)$ ways in which the $2 k$ indices may be paired up. Hence

$$
\sum_{\substack{p_{1}, \ldots, p_{2 k} \leq y \\ p_{1} \ldots p_{2 k}=\square}} \frac{\left|a\left(p_{1}\right) \ldots a\left(p_{2 k}\right)\right|}{\sqrt{p_{1} \ldots p_{2 k}}} \leq \frac{(2 k) !}{k ! 2^{k}}\left(\sum_{p} \frac{|a(p)|^{2}}{p}\right)^{k}
$$

Proof of Proposition 6.2. For brevity put $\mathcal{V}=\mathcal{V}\left(z_{1}, z_{2}, X\right)$, and set

$$
A= \begin{cases}\frac{1}{2} \log \log \log X, & V \leq \mathcal{V}, \\ \frac{\mathcal{V}}{2 V} \log \log \log X, & \mathcal{V}<V \leq \frac{1}{16} \mathcal{V} \log \log \log X, \\ 8, & V>\frac{1}{16} \mathcal{V} \log \log \log X\end{cases}
$$

Define further $x=X^{A / V}$ and $z=x^{1 / \log \log X}$.

By taking $x=\log X$ in 6.5 and bounding the sum over $p$ trivially, we may assume $V \leq 5 \log X / \log \log X$. Then by 6.5 we have

$$
\log \left|L\left(1 / 2+z_{1}, f \otimes \chi_{d}\right) L\left(1 / 2+z_{2}, f \otimes \chi_{d}\right)\right| \leq S_{1}+S_{2}+\mathcal{M}\left(z_{1}, z_{2}, X\right)+5 \frac{V}{A},
$$

where $S_{1}$ is the sum there truncated to $p \leq z$, and $S_{2}$ is the sum over $z<p \leq x$. If $d$ is such that $\log \left|L\left(1 / 2+z_{1}, f \otimes \chi_{d}\right) L\left(1 / 2+z_{2}, f \otimes \chi_{d}\right)\right| \geq V+\mathcal{M}\left(z_{1}, z_{2}, X\right)$, then either

$$
S_{2} \geq V / A, \quad \text { or } \quad S_{1} \geq V(1-6 / A)=: V_{1} .
$$


By Lemma 6.3 we see that for any $k \leq V /(2 A)-1$ we have

$$
\sum_{|d| \leq X}^{b}\left|S_{2}\right|^{2 k} \ll X \frac{(2 k) !}{k ! 2^{k}}\left(\sum_{z<p \leq x} \frac{4}{p}\right)^{k} \ll X(3 k \log \log \log X)^{k} .
$$

Hence, choosing $k=\lfloor V /(2 A)\rfloor-1$ and with a little calculation, the number of discriminants $|d| \leq X$ with $S_{2} \geq V / A$ is

$$
\ll X \exp \left(-\frac{V}{4 A} \log V\right) .
$$

We now seek a bound for the number of discriminants with $S_{1}$ large. By Lemma 6.3 we find that for any $k \leq \log \left(X^{1 / 2} / \log X\right) / \log z$,

$$
\sum_{|d| \leq X}^{b}\left|S_{1}\right|^{2 k} \ll X \frac{(2 k) !}{k ! 2^{k}}\left(\sum_{p \leq z} \frac{a(p)^{2}}{p}\right)^{k}
$$

where $a(p)=\lambda_{f}(p) p^{-\lambda_{0} / \log x} \frac{\log (x / p)}{\log x} \operatorname{Re}\left(p^{-z_{1}}+p^{-z_{2}}\right)$. Note that

$$
\begin{aligned}
\sum_{p \leq z} \frac{a(p)^{2}}{p} & \leq \frac{1}{4} \sum_{p \leq X} \frac{\lambda_{f}(p)^{2}}{p}\left(p^{-z_{1}}+p^{-\overline{z_{1}}}+p^{-z_{2}}+p^{-\overline{z_{2}}}\right)^{2} \\
& =\mathcal{V}\left(z_{1}, z_{2}, X\right)+O(\log \log \log X),
\end{aligned}
$$

upon using (6.3) and (6.4) and partial summation. Thus the number of $|d| \leq X$ such that $S_{1} \geq V_{1}$ is

$$
\begin{aligned}
& \ll X V_{1}^{-2 k} \frac{(2 k) !}{2^{k} k !}\left(\mathcal{V}\left(z_{1}, z_{2}, X\right)+O(\log \log \log X)\right)^{k} \\
& \ll X\left(\frac{2 k\left(\mathcal{V}\left(z_{1}, z_{2}, X\right)+O(\log \log \log X)\right)}{e V_{1}^{2}}\right)^{k} .
\end{aligned}
$$

When $V \leq(\log \log X)^{2}$, we take $k$ to be $\left\lfloor V_{1}^{2} /\left(2 \mathcal{V}\left(z_{1}, z_{2}, X\right)\right)\right\rfloor$, and for $V>(\log \log X)^{2}$ we take $k$ to be $\lfloor 10 V\rfloor$. Then the above estimates give that the number of discriminants $|d| \leq X$ with $S_{1} \geq V_{1}$ is

$$
\ll X \exp \left(-\frac{V_{1}^{2}}{2 \mathcal{V}\left(z_{1}, z_{2}, X\right)}\left(1+O\left(\frac{\log \log \log X}{\log \log X}\right)\right)\right)+X \exp (-V \log V) .
$$

Combining this estimate with our estimate for the frequency with which $S_{2}$ can be large, we obtain the proposition.

Acknowledgments. The authors are partially supported by grants from the NSF (DMS-0500711 and DMS-0758235).

\section{References}

[1] Bucur, A., Diaconu, A.: Moments of quadratic Dirichlet $L$-functions over rational function fields. Preprint 
[2] Bump, D., Friedberg, S., Hoffstein, J.: Nonvanishing theorems for $L$-functions of modular forms and their derivatives. Invent. Math. 102, 543-618 (1990) Zbl 0721.11023 MR 1074487

[3] Chandee, V.: On the correlation of shifted values of the Riemann zeta function. Preprint

[4] Conrey, J. B., Farmer, D.: Mean values of $L$-functions and symmetry. Int. Math. Res. Notices 2000, no. 17, 883-908 Zbl 1035.11038 MR 1784410

[5] Conrey, J. B., Farmer, D., Keating, J., Rubinstein, M., Snaith, N.: Integral moments of Lfunctions. Proc. London Math. Soc. (3) 91, 33-104 (2005) Zbl 1075.11058 MR 2149530

[6] Davenport, H.: Multiplicative Number Theory. 3rd ed., Grad. Texts in Math. 74, Springer, New York (2000) Zbl 1002.11001 MR 1790423

[7] Erdélyi, A., Magnus, W., Oberhettinger, F., Tricomi, F. G.: Tables of Integral Transforms. Vol. I. McGraw-Hill, New York (1954) Zbl 0055.36401 MR 0061695

[8] Gradshteyn, I. S., Ryzhik, I. M.: Tables of Integrals, Series, and Products. 6th ed., Academic Press, San Diego, CA (2000) Zbl 0981.65001 MR 1773820

[9] Heath-Brown, D. R.: A mean value estimate for real character sums. Acta Arith. 72, 235-275 (1995) Zbl 0828.11040 MR 1347489

[10] Iwaniec, H.: On the order of vanishing of modular $L$-functions at the critical point. Sém. Théor. Nombres Bordeaux (2) 2, 365-376 (1990) Zbl 0719.11029 MR 1081731

[11] Iwaniec, H., Kowalski, E.: Analytic Number Theory. Amer. Math. Soc. Colloq. Publ. 53, Amer. Math. Soc., Providence, RI (2004) Zbl 1059.11001 MR 2061214

[12] Keating, J. P., Snaith, N. C.: Random matrix theory and $L$-functions at $s=1 / 2$. Comm. Math. Phys. 214, 91-110 (2000) Zbl 1051.11047 MR 1794267

[13] Murty, M. R., Murty, V. K.: Mean values of derivatives of modular $L$-series. Ann. of Math. (2) 133, 447-475 (1991) Zbl 0745.11032 MR 1109350

[14] Rudnick, Z., Soundararajan, K.: Lower bounds for moments of $L$-functions. Proc. Nat. Acad. Sci. USA 102, 6837-6838 (2005) Zbl 1159.11317 MR 2144738

[15] Rudnick, Z., Soundararajan, K.: Lower bounds for moments of $L$-functions: symplectic and orthogonal examples. In: Multiple Dirichlet Series, Automorphic Forms, and Analytic Number Theory, Proc. Sympos. Pure Math. 75, Amer. Math. Soc., Providence, RI, 293-303 (2006) Zbl 1120.11039 MR 2279944

[16] Soundararajan, K.: The fourth moment of Dirichlet $L$-functions. In: Analytic Number Theory, Clay Math. Proc. 7, Amer. Math. Soc., Providence, RI, 239-246 (2007) Zbl pre05233966 MR 2362204

[17] Soundararajan, K.: Moments of the Riemann zeta-function. Ann. of Math. 170, 981-993 (2009) Zbl pre05610438 MR 2552116

[18] Soundararajan, K.: Nonvanishing of quadratic Dirichlet $L$-functions at $s=1 / 2$. Ann. of Math. (2) 152, 447-488 (2000) Zbl 0964.11034 MR 1804529

[19] Titchmarsh, E. C.: The Theory of the Riemann Zeta-Function. 2nd ed., Oxford Univ. Press, New York (1986) Zbl 0601.10026 MR 0882550 